\title{
THE UNIVERSE AT LOW RADIO FREQUENCIES
}

Edited by: A. PRAMESH RAO, G. SWARUP AND GOPAL-KRISHNA
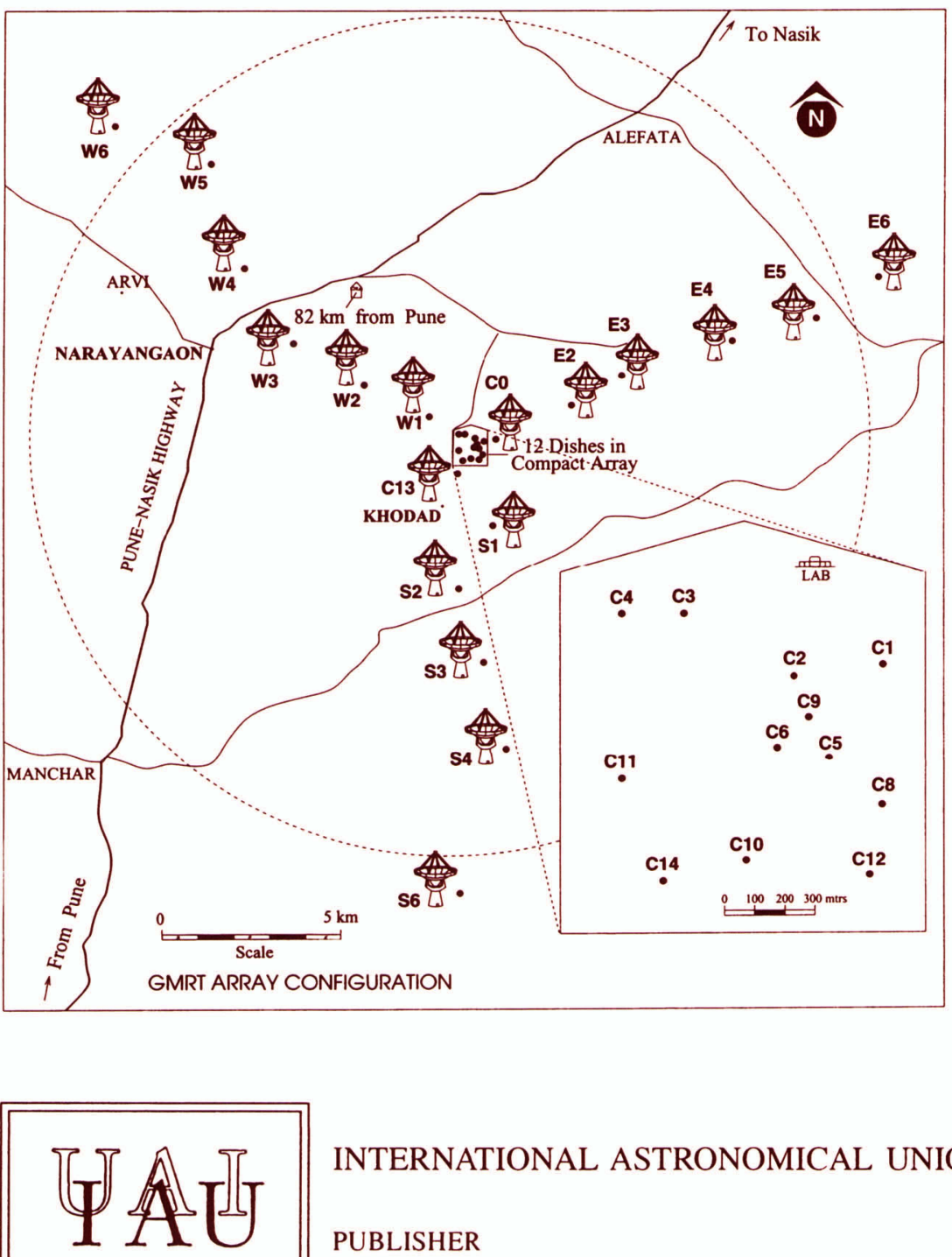

INTERNATIONAL ASTRONOMICAL UNION

PUBLISHER

THE ASTRONOMICAL SOCIETY OF THE PACIFIC 
IAU SYMPOSIUM VOLUME 199

Configuration of the Giant Metrewave Radio Telescope (GMRT) taken from the contribution of Rao in this volume. The GMRT is the first of a number of low frequency radio telescopes being planned around the world. 


\section{THE ASTRONOMICAL SOCIETY OF THE PACIFIC}

390 Ashton Avenue - San Francisco - California - USA 94112-1722

Phone: (415) 337-1100 E-Mail: catalog@astrosociety.org

Fax: (415) 337-5205

Web Site: www.astrosociety.org

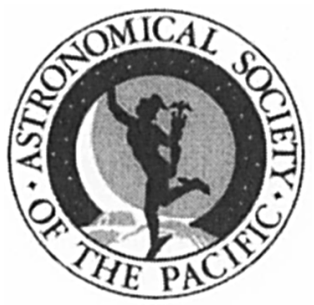

ASP CONFERENCE SERIES - EDITORIAL STAFF

Managing Editor: D. H. McNamara

Associate Managing Editor: J. W. Moody
LaTeX-Computer Consultant: T. J. Mahoney

Production Manager: Enid L. Livingston

Production Assistant: Andrea Weaver:

PO Box 24463 - 211-KMB - Brigham Young University - Provo - Utah 84602-4463

Phone: (801) 422-2111 Fax: (801) 378-4049 E-Mail: pasp@byu.edu

ASP CONFERENCE SERIES PUBLICATION COMMITTEE:

$\begin{array}{ll}\text { Alexei V. Filippenko } & \text { Geoffrey Marcy } \\ \text { Ray Norris } & \text { Donald Terndrup } \\ \text { Frank X. Timmes } & \text { C. Megan Urry }\end{array}$

A listing of all other IAU Volumes published by the ASP is cited at the back of this volume 


\title{
INTERNATIONAL ASTRONOMICAL UNION \\ 98bis, Bd Arago - F-75014 Paris - France \\ Tel: +33143258358 E-mail: iau@iap.fr \\ Fax: +33 143252616 Web Site: www.iau.org
}

\section{THE UNIVERSE AT LOW RADIO FREQUENCIES}

\author{
Proceedings of the $199^{\text {th }}$ Symposium \\ of the International Astronomical Union \\ held in Pune, India \\ 30 November-4 December 1999
}

Edited by

A. Pramesh Rao

National Centre for Radio Astrophysics (TIFR), Pune, India

G. Swarup

National Centre for Radio Astrophysics (TIFR), Pune, India

and

\section{Gopal-Krishna}

National Centre for Radio Astrophysics (TIFR), Pune, India 
C 2002 by International Astronomical Union All Rights Reserved

No part of the material protected by this copyright notice may be reproduced or utilized in any form or by any means - graphic, electronic, or mechanical including photocopying, taping, recording or by any information storage and retrieval system, without written permission from the IAU.

Library of Congress Cataloging in Publication Data

Main entry under title

LOC \#: 2002112081

ISBN: $1-58381-121-4$

IAU Publications - First Edition

Published on behalf of IAU by: The Astronomical Society of the Pacific

Printed in United States of America by: Sheridan Books, Chelsea, Michigan 


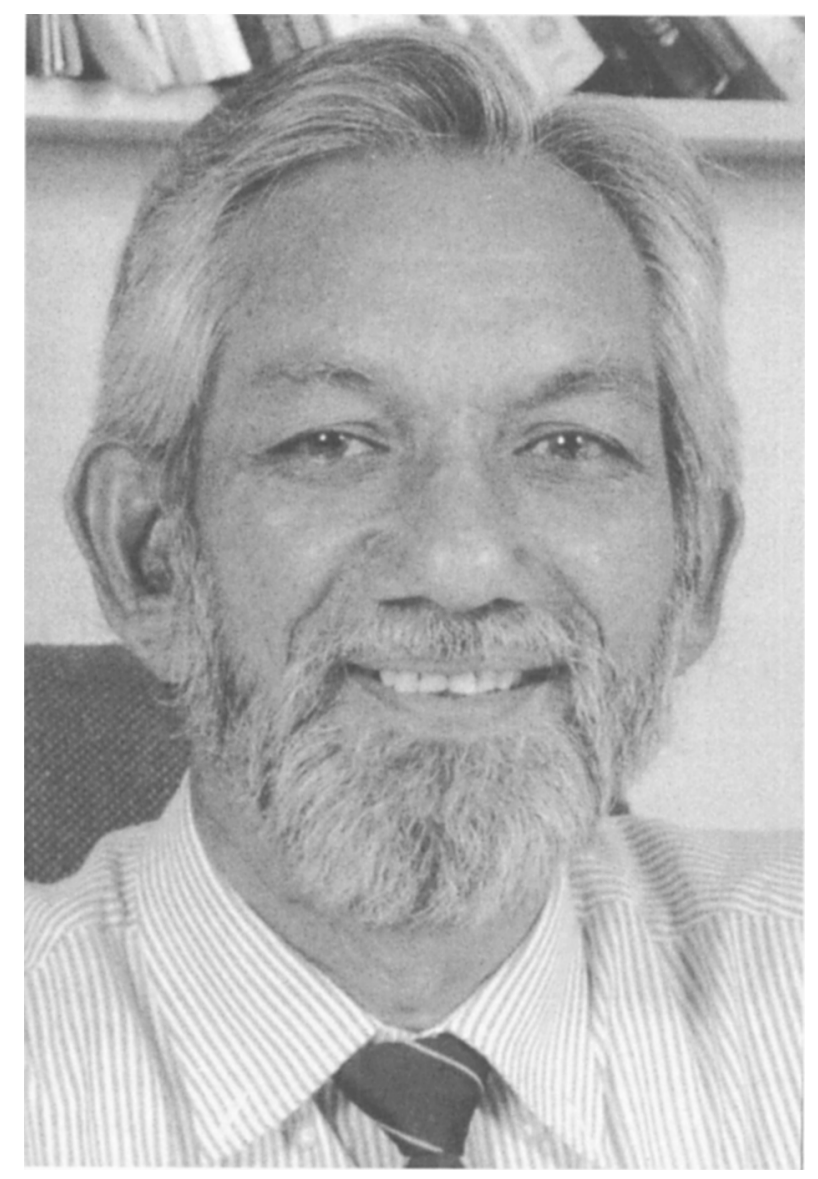

We dedicate this volume to

\section{VIJAY KUMAR KAPAHI 1944-1999}




\section{Table of Contents}

Preface ..................... $\mathrm{xv}$

List of Participants ................. . . xix

Conference Photo .................. xxiii

\section{Part 1: Radio Source Surveys and Cosmology}

Radio Source Surveys $\ldots \ldots \ldots \ldots \ldots$

J.J. Condon

The Sydney University Molonglo Sky Survey (SUMSS) and Optical

Redshift Surveys of the Southern Sky . . . . . . . . . 11

E.M. Sadler and R.W. Hunstead

Cambridge Low Frequency Surveys . . . . . . . . . . . . . . 21

D.A. Green

A Low-frequency Southern Sky Survey Using the Mauritius Radio

Telescope . . . . . . . . . . . . . . . 25

N. Udaya Shankar

WSRT $1.4 \mathrm{GHz}$ Observations of the Hubble Deep Field . . . . . . . 32

M.A. Garrett, G. de Bruyn, W. Baan and R.T. Schilizzi

Cosmological Studies from Radio Source Samples . . . . . . . . . . 34

S. Rawlings

Radio Source Evolution Derived from Low Frequency Surveys . . . . 50

C.A. Jackson and J.V. Wall

Large Scale Structure Among $z \sim 2$ Quasars as a Cosmological

Standard Ruler . . . . . . . . . . . . . . . . 54

B.F. Roukema and G.A. Mamon

The Luminosity Periodicity of Galaxies and Quasars in the Decametric Range ..................... 56

A.P. Miroshnichenko

Cosmic Microwave Background at Low Frequencies . . . . . . . . 58

R. Subrahmanyan

Absorption Against the Cosmic $2.7 \mathrm{~K}$ Background . . . . . . . . . 66

S. Chandra and W.H. Kegal

\section{Part 2: Extragalactic Neutral Hydrogen and Cosmology}

Signatures of HI in the Early Universe: The End of the Dark Ages . . 71 A. Meiksin

A Step to the Reionization Epoch

P.A. Shaver 
$21 \mathrm{~cm}$ Absorption Lines at High Redshift from Intervening Galaxies . 83 F.H. Briggs

Associated HI in Absorbers at High Redshift . . . . . . . . . . 91

R.C. Vermeulen

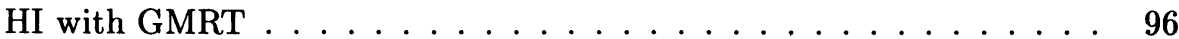

J.N. Chengalur

A WSRT Survey for HI Absorption at Moderate Redshifts . . . . . . . 102 W.M. Lane and F.H. Briggs

Probing HI in the Universe with SKA . . . . . . . . . . . 106 J.M. van der Hulst

Probing Large Scale Structures in HI with GMRT . . . . . . . . . . 108

S. Bharadwaj, B.B. Nath and S.K. Sethi

Probes of Low Surface Brightness Galaxies through Low Frequency

Spectroscopy with GMRT . . . . . . . . . . . . . 110

D. Narasimha and S.M. Chitre

Using Gravitational Lenses to Probe HI at High z . . . . . . . . . . 114

T.D. Saini, S.K. Sethi and S. Bharadwaj

Search for Radio Recombination Lines towards the Gravitational Lens

PKS1830-211 . . . . . . . . . . . . . . . 116

N.R. Mohan, K.R. Anantharamaiah and W.M. Goss

HI absorption in Radio Galaxies . . . . . . . . . . . . . . . 118

R. Morganti, T.A. Oosterloo, G. van Moorsel, C.N. Tadhunter and $N$. Killeen

Atomic Hydrogen Gas Images of QSO Host Galaxies . . . . . . . 122 J. Lim and P.T.P. Ho

An HI Search for the Host Galaxies of 27 Radio-loud AGN at z 2.3 . 127 T.Ghosh, M.M. Davis, C.J. Salter and M.C. Aller

HI in Active Galactic Nuclei . . . . . . . . . . . . . . . . . . . . . . 129

Z. Yu and D. Jiang

\section{Part 3: Clusters of Galaxies}

Observational Properties of Diffuse Halos in Clusters . . . . . . . . 133 L. Feretti

Theoretical Implications of Diffuse Non-Thermal Emission from

Clusters of Galaxies . . . . . . . . . . . . . . . . . . 141

T.A. Enßlin

Radio Halos and Relics in Clusters of Galaxies and Detection Statistics 149

G. Giovannini, L. Feretti and F. Govoni

Diffuse Sources in Clusters: What Turns them on . . . . . . . . . . 151

T. Murphy and R.W. Hunstead

Extreme Relic Radio Sources in Four Southern Clusters . . . . . . . 153

H. Andernach, O.B. Slee, A.L. Roy and M.Ehle 
The Dual Radio Relics of A3667 . . . . . . . . . . . . . . 157

M. Johnston-Hollitt, R. W. Clay, R. D. Ekers, M. H. Wieringa and R. W. Hunstead

A Large Diffuse Radio Source in a Cluster of Galaxies at $\mathrm{z}=0.13 \ldots 159$

Gopal-Krishna, V.K. Kulkarni, J. Bagchi and J. Melnick

Active Galaxies and Candidate Remnants in the Core of the Shapley

Concentration . . . . . . . . . . . . . . . 161

T. Venturi, S. Bardelli, D. Dallacasa, R.W. Hunstead,

R. Morganti and T. Tzioumis

Environmental Effects and the Dynamical State of Coma from a VLA

HI Survey . . . . . . . . . . . . . . . 163

H. Bravo-Alfaro, J. H. van Gorkom, V. Cayatte and C. Balkowski

Coma Southwest - as seen by the GMRT . . . . . . . . . . . . 166

K.S. Dwarakanath and J.N. Chengalur

\section{Part 4: Extragalactic Radio Sources}

Extended Extragalactic Radio Emission . . . . . . . . . . . . . 171

F.N. Owen, M.J. Ledlow, J.A. Eilek, N.E. Kassim, N. Miller,

K.S. Dwarakanath and R.J. Ivison

Spectral Mapping of Classical Double Radio Sources . . . . . . . . . . 179 J.P. Leahy and T.W.B. Muxlow

3C Radio Sources as They've Never Been Seen Before . . . . . . . . 189

K.M. Blundell, N.E. Kassim and R.A. Perley

VLA Images of Two Extended Radio Galaxies . . . . . . . . . . . 193

W. Junor, F. Mantovani, R. Morganti and L. Padrielli

Giant Radio Galaxies and the Inter Galactic Medium . . . . . . . 195

A.P. Schoenmakers, A.G. de Bruyn, H.J.A. Röttgering and $H$. van der Laan

Giant Radio Sources: Evolution and GMRT Observations . . . . . . . 199

C.H. Ishwar-Chandra and D.J. Saikia

Statistics of Giant Radio Sources . . . . . . . . . . . . . . 203

J. Machalski and M. Jamrozy

Statistical Study on Large Samples of Radio Sources . . . . . . . 207

X.Z. Zhang, B. Peng and P.C. Chen

Compact Steep Spectrum Radio Sources . . . . . . . . . . . . 209

S. Jeyakumar and D.J. Saikia

Physical Conditions in CSS Radio Sources . . . . . . . . . . . . 211

S.A. Tyul'bashev and P.A. Tchernikov 
Interferometer Observations of Extragalactic Radio Sources at

Decameter Wavelengths . . . . . . . . . . . . . . 213

A.V. Megn, S.Ya. Braude, S.L. Rashkovsky, V.A. Shepelev,

N.K.Sharykin and G.A. Inyutin

The Problem of Identifying Decametric Sources . . . . . . . . . 215

O.V. Verkhodanov, H. Andernach and N.V. Verkhodanova

Radio-optical Identification of Very-Steep Spectrum Radio Sources

from the UTR-2 Catalogue . . . . . . . . . . . . . . 217

H. Andernach, O.V. Verkhodanov and N.V. Verkhodanova

BVRI-Photometry of Distant Radio Galaxies from RC Catalogue in

SAO RAS . . . . . . . . . . . . . . . . . 219

Yu.N. Parijski, W.M. Goss, A.I. Kopylov, N.S. Soboleva,

O.V. Verkhodanov, A.V. Temirova and O.P.Zhelenkova

Study of Objects of Low Radio Frequency Catalogues and IRAS Data

- Cross-Identification . . . . . . . . . . . . . . . 221

O.V. Verkhodanov and S.A. Trushkin

The Nuclear Structure of the Giant Radio Galaxy, 3C236 . . . . . . 225

W.W. Tian, R.T. Schilizzi and R. Nan

Extended X-ray Emission from FRIIs and RL Quasars . . . . . . 227

G. Setti, G. Brunetti and A Comastri

Effects of Synchrotron Loss on the Low-Frequency Spectra of

Extragalactic Radio Sources with Inhomogeneities . . . . . . . 231

N. Tsvyk

Outflows and the Disk-Halo Connection in Galaxies . . . . . . . 233

J.A. Irwin

GMRT Observations of M 82 and NGC $3079 \ldots \ldots$. . . . . . . 241

J.A. Irwin and D.J. Saikia

Radio Recombination Lines from Starburst Galaxies: High and Low

Density Ionized Gas . . . . . . . . . . . . . . . . . 243

N.R. Mohan, K.R. Anantharamaiah and W.M. Goss

Low Frequency Catalogues of the CATS Database . . . . . . . . . . 245

O.V. Verkhodanov, S.A. Trushkin and H. Andernach

The SEDs Database to Study Evolution of Radio Galaxies . . . . . 247

O.V. Verkhodanov, A.I. Kopylov, O.P. Zhelenkova,

N.V. Verkhodanova, V.N. Chernenkov, Yu.N. Parijskij,

N.S. Soboleva and A.V. Temirova

\section{Part 5: Galactic Surveys and Extended Emission}

Galactic Plane Surveys at Low Frequencies . . . . . . . . . . 251

A.R. Taylor

The Molonglo Galactic Plane Survey: MGPS2 _ . . . . . . . . 259

A.J. Green 
Radio Continuum Surveys of the Galaxy and Galaxies . . . . . . . . 262 R. Wielebinski

The Galactic Center at $327 \mathrm{MHz}$. . . . . . . . . . . . . . . 268

T.N. LaRosa, N.E. Kassim, T.J.W. Lazio and S.D. Hyman

VLA Observations of the Galactic Center at $74 \mathrm{MHz}$. . . . . . . . 272

K.R. Anantharamaiah, N.E. Kassim, T.J.W. Lazio, W.M. Goss and H. Falcke

GMRT Observations of the Galactic Centre region . . . . . . . . . 274 S. Roy and A.P. Rao

Radio Observations of Galactic SNRs . . . . . . . . . . . . . . 276 D.A. Green

Radio Observations of Supernova Remnants and the Surrounding

Interstellar Medium . . . . . . . . . . . . . . . . 284 G. Dubner

Low Frequency Insights into Supernova Remnants . . . . . . . . . 291

K.K. Dyer, S.P. Reynolds, K.J. Borkowski N.E. Kassim and C.K. Lacey

Radio Spectra of Complete Sample of Galactic Supernova Remnants . 295 S.A. Trushkin

On the Association of G343.1-2.3 and PSR 1706-44 . . . . . . . . . . 299

R. Dodson, K. Golap, J. Osborne and N. UdayaShankar

The Radio Spectral Index of $3 \mathrm{C58}$. . . . . . . . . . . . . . . . . 303

M.F. Bietenholz, N. Kassim and K. Weiler

Deep Imaging of SNRs at Low Frequencies Using the GMRT . . . . . 307 S. Bhatnagar

A Multifrequency Radio Spectral Study of SNR HB21 . . . . . . . . . 309

X.Z. Zhang, L.A. Higgs, T.L. Landecker, S.J. Qian and $X . J W u$

Supernova Remnant G11.2-0.3 and the ISM . . . . . . . . . . . . 311 A.C. Seth

Multifrequency GMRT observations of HII regions . . . . . . . . . 313 A. Omar, J.N. Chengalur and D.A. Roshi

Anisotropy of Hectometric Cosmic Background . . . . . . . . . . . . 315

Y.V. Tokarev, M.L. Kaiser, G.N. Boiko and P.V. Gustov

\section{Part 6: Spectral Studies of our Galaxy}

Low frequency Recombination Lines of Hydrogen . . . . . . . . . . 319 K.R. Anantharamaiah

Low frequency Carbon Recombination Lines . . . . . . . . . . . 327 A.A. Konovalenko

A Study of Low density Ionized Gas in the Galactic Plane . . . . . . 335 D.A. Roshi and K.R. Anantharamaiah 
A Galactic Plane Survey in the CO (2-1) Line with the $60 \mathrm{~cm}$

Telescopes to Address Physical Condition of the Interstellar

Matter ...................... 339

T. Handa, T. Hasegawa, J.I. Morino, T. Sawada, S. Sakamoto, K.S. Usuda, A. Luna, L. Bronfman and M. Hayashi

HI $21 \mathrm{~cm}$-line Observations with the GMRT Towards Interstellar

Clouds Previously Seen in Optical Absorption . . . . . . . . . . 341

R. Mohan, K.S. Dwarakanath, G. Srinivasan and J.N. Chengalur

Preliminary Results of Galactic Radio Recombination Line

Observations using the GMRT . . . . . . . . . . . . . . 343

N.G. Kantharia and D.A. Roshi

Galactic Carbon Recombination Lines near $327 \mathrm{MHz}$. . . . . . . . 345

D.A. Roshi, N.G. Kantharia and K.R. Anantharamaiah

Carbon Recombination Lines at $34.5 \mathrm{MHz}$ from the Galactic Plane . 347 N.G. Kantharia and K.R. Anantharamaiah

Recombination Radio Lines at Very Low Frequencies . . . . . . . . 349

A.A. Konovalenko, S.V. Stepkin and D.V. Shalunov

Populations of Hydrogen-like Atoms or Ions and Radio Recombination

Lines (RRL's) Interpretation . . . . . . . . . . . . . . 351

N.I. Rovenskaya

\section{Part 7: Pulsars and other Compact Galactic Objects}

Pulsars: An Observational Overview . . . . . . . . . . . . . 355

R.N. Manchester

Pulsars and the ISM . . . . . . . . . . . . . . . 363

Y. Gupta

First Results from Simultaneous Dual Frequency Observations

of Pulsars . . . . . . . . . . . . . . . . . 369

Y. Gupta, P. Gothoskar and N.D.R. Bhat

Detection of New Emission Components in PSR B0329+54 . . . . . . 373

R.T. Gangadhara, Y. Gupta and D.R. Lorimer

Orthogonal Polarization Modes from PSR B0301+19 and B0355+54 . 375

R.T. Gangadhara

Low-frequency Emission Regions in Pulsars . . . . . . . . . . . . 377

J. Kijak

Low-frequency Profiles of the Crab Pulsar . . . . . . . . . . 379

A. Kuzmin

Giant Pulses from Two Pulsars . . . . . . . . . . . . . . . . . 381

A.K. Singal, P.K. Manoharan and R.G. Strom

The NFRA Pulsar Machine PuMA . . . . . . . . . . 383

R.G. Strom 
A WSRT Search for Millisecond Pulsars . . . . . . . . . . . . 387

W.W. Tian, R. G. Strom, B. W. Stappers, X. Z. Zhang,

$X$. J. Wu and R. Ramachandran

Low-frequency Observations of Millisecond Pulsars . . . . . . . . . . 389

A. Kuzmin

Unique Radio Pulsar Geminga . . . . . . . . . . . . . . . 393

V.M. Malofeev and O.I. Malov

A VLA search for the Geminga Pulsar at 74 and $326 \mathrm{MHz} \ldots \ldots . .395$

T.J.W. Lazio and N.E. Kassim

Radio Variability of the Galactic X-ray Binaries with

Relativistic Jets . . . . . . . . . . . . . . . . . . 397

S.A. Trushkin and N.N. Bursov

New Radiation Formulae of Relativistic Electrons in Curved Magnetic

Field Lines . . . . . . . . . . . . . . . . . . . 400

Ya.M. Sobolev

Frequency Spectra Fluctuations in the Radio Interferometry of

Polarised Radiation . . . . . . . . . . . . . . . . . 402

M.R. Olyak

\section{Part 8: Sun and Planetary Systems}

Low Frequency Planetary Radio Astronomy . . . . . . . . . . . 407 R.J. Sault

Solar Radio Astronomy at Low Frequencies . . . . . . . . . . . . 415 M. Pick

Radio Astronomical Scintillation in the Solar Wind Plasma: Imaging Interplanetary Disturbances . . . . . . . . . . . . 426

P.K. Manoharan, M. Pick and LASCO Consortium

Solar Observation with Miyun Radio Telescope . . . . . . . . . . . . 430

X.Z. Zhang, T.Y. Piao, L.S.Kang and L. Pang

Observations of Solar Bursts Using the New Radio Spectrograph . . . 432

A. Shanmugaraju, S. Umapathy and V. Balasubramanian

Interpretation of James' Experiments in Plasma Theory of Solar

Radar Echoes . . . . . . . . . . . . . . . . . . . . . . 434

V.N. Mel'nik

Part 9: Instrumentation and Techniques

GMRT - Current Status . . . . . . . . . . . . . . 439

A.P. Rao

The $74 \mathrm{MHz}$ System on the VLA . . . . . . . . . . . . . 447

R. A. Perley, W.C. Erickson and N.E. Kassim 
Practical Lessons from Low Frequency Imaging with the VLA . . . . 455 C.K. Lacey and N.E. Kassim

Low Frequency Science with the Square Kilometre Array . . . . . . 459 A.R. Taylor

Concepts and Technical Studies of the Square Kilometre Array . . . . 467 A. van Ardenne

The Low-Frequency Array (LOFAR): Opening a New Window on the Universe . . . . . . . . . . . . . . . . . . . . 474

N.E. Kassim, T. J.W. Lazio, W.C. Erickson, P.C. Crane,

R.A. Perley and B. Hicks

Towards a Concept Design for a LOFAR . . . . . . . . . . . . . 484 J.D. Bregman

ALOFT: A Potential Low Frequency Space VLBI Mission . . . . . . 486

H. Hirabayashi, I.M. Avruch and D.W. Murphy

Low Frequency Radio Astronomy from Above the Ionosphere . . . . 488 D.L. Jones

The Universe at Very Low Radio Frequencies . . . . . . . . . . 490

S.Ya. Braude, A. A. Konovalenko and A. V. Megn

Low Frequency VLBI Project . . . . . . . . . . . . . . . . . . . 492

I.E. Molotov, S.F. Likhachev, A.A. Chuprikov, A. Dementiev,

B. Lipatov, M. Nechaeva, S. Snegirev, N. Dugin,

S. Ananthakrishnan, V. Balasubramanian, A. Benz,

F. Mantovani, X. Liu, X. Hong, A. Kus, E.P. Molotov,

S.P. Ignatov, B.A. Poperechenko, Y.N. Gorshenkov

and A.A. Konovalenko

The Development of the FAST Project in China . . . . . . . . . 494

R. Nan, B. Peng, Y. Qiu, L. Zhu, Y. Su and W. Zhu

Radio Frequency Interference . . . . . . . . . . . . . . . . 498

R. D. Ekers and J. F. Bell

High dynamic range, Interferences Tolerant, Digital Receivers for

Radioastronomy: Results and Projects at Paris

and Nanay Observatory . . . . . . . . . . . . . . 506

C. Rosolen, A. Lecacheux, E. Gerard, V. Clerc and L. Denis

Wide Field Imaging at Low Frequencies . . . . . . . . . . . 508

R.J. Sault

Filter CLEAN - An Improved Method for CLEANing Images . . . . 512

A. McPhail

Geometric Phase in Phasing of Antenna Arrays . . . . . . . . . . . . 514

R. Bhandari

Author Index $\ldots \ldots \ldots \ldots \ldots \ldots \ldots \ldots \ldots \ldots$ 